\title{
Combining Extended Kalman Filter with Complementary Filter for UAV Attitude Estimation based on MEMS MARG Sensors
}

\author{
Xiang REN ${ }^{1, a}$, Shoubin LIU $^{2, b}$ * \\ ${ }^{1,2}$ Department of Mechanical Engineering and Automation, Harbin Institute of Technology \\ Shenzhen Graduate School, Shenzhen, 518055, China

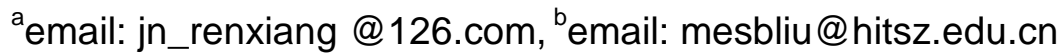 \\ ${ }^{*}$ Corresponding author
}

\begin{abstract}
Keywords: attitude estimation, data fusion, MEMS sensors, EKF, complementary filter.
Abstract. Reliable attitude information is desired for navigation and control of rotor unmanned aerial vehicles (UAV). Rotor UAV's attitude can be determined by fusing redundant data from MEMS MARG (Magnetic, Angular Rate, and Gravity) sensors with fusion techniques. The extended Kalman filter (EKF)-based fusion algorithms are commonly adopted. However, there exists a contradiction between convergence speed and noise suppression in EKF-based algorithms. This paper presents a novel fusion algorithm combining EKF with complementary filter to estimate the attitude of rotor UAV. Firstly, gyros' measurements of angular rates are corrected by measurements of accelerometers with a Mahony passive complementary filter. The corrected angular rates as well as the measurements of accelerometers and magnetometers are then input to an EKF to implement data fusion. Results of validation experiments show that the proposed fusion method can generate attitude angles accurately and fuse multi-sensor data efficiently.
\end{abstract}

\section{Introduction}

A rotor UAV desires an inexpensive and small attitude and heading reference system (AHRS) to determine its attitude. The low-cost AHRS can be constructed with MEMS MARG sensors which contain triaxial gyros, triaxial accelerometers and triaxial magnetometers [1,2]. Because a MEMS gyro has a large drift and unneglectable static bias, its attitude information after long hours is not accurate. Similarly, outputs of accelerometers and magnetometers are not reliable since accelerometers are susceptible to the impact of vibration, acceleration and deceleration, while the magnetometers are susceptible to the impact of magnetic noise. As a result, the MEMS MARG sensors cannot be used separately. Outputs of triaxial accelerometers, triaxial magnetometers and triaxial gyros have to be integrated to form a reliable attitude output of rotor UAV [3,4]. Many algorithms are proposed for the fusion of redundant multi-sensor data [5-7].

A digital filter is usually used for removing the noise and disturbs from an input signal in a single-input and single-output system. Some digital filters, such as extended Kalman filter (EKF) and complementary filter, have a signal fusion ability in a multiple-input and multiple-output system $[8,9]$. The EKF-based fusion algorithms are thus commonly used for data fusion. However, there exists a contradiction between convergence speed and noise suppression in EKF-based algorithms. This paper presents a new fusion method combing extended Kalman filter with Mahony passive complementary filter. Firstly, gyros' measurements of angular rates are corrected by measurements of accelerometers with a Mahony passive complementary filter. The corrected angular rates as well as the measurements of accelerometers and magnetometers are then input to an EKF to implement data fusion. Finally, validation experiments are implemented to prove the effectiveness of the presented algorithm.

\section{Complementary Filter}

The classical complementary filter is composed of a high pass filter and a low pass filter. It has a good effect for fusing high bandwidth position measurements and low bandwidth rate measurements of the first order kinematic systems. For a position variable $x$, its rate variable $v$ can be expressed as $v=\delta$, 
and its measurements $y_{x}$ can be expressed as $y_{x}=x+\mu_{x}$ where $\mu_{x}$ is the high frequency noise. For the rate variable $v$, its measurements $y_{v}$ can be expressed as $y_{v}=v+\mu_{v}+b(t)$ where $\mu_{v}$ is the low frequency noise and $b(t)$ is the static bias varying with time.

Fig. 1 shows a block diagram of a classical complementary filter to fuse the position measurements $y_{x}$ with the rate measurements $y_{v}$, where $C(s)$ is a compensator. According to the principle of superposition, the fusing result $\hat{x}$ is written in the form of

$$
\begin{aligned}
\hat{x} & =\frac{s}{s+C(s)} \frac{y_{v}}{s}+\frac{C(s)}{s+C(s)} y_{x} \\
& =F(s) \frac{y_{v}}{s}+G(s) y_{x}
\end{aligned}
$$

where $F(s)$ represents a high pass filter, $G(s)$ represents a low pass filter, and $F(s)+G(s)=1$. In general, the overall filter is considered covering the whole frequency domain. If $C(s)=k_{P}, s=j \omega$, then $F(s)$ and $G(s)$ can be written as $1 /\left(1-j k_{p} / \omega\right)$ and $1 /\left(1+j \omega / k_{p}\right)$ where $\omega$ represents input angular frequency. The crossover frequency of the high pass filter and the low pass filter is $k_{p} / 2 \pi$.

An unsolved problem of the classical complementary filter is the static bias $b(t)$. Because of the effect of integral $y_{v} / s$, the error caused by static bias will be accumulated. If the static bias $b(t)$ is assumed to be a constant $\mathrm{b}_{0}$, it is possible to add an integrator into the compensator $C(s)$ to eliminate the error. This results a so-called Mahony passive complementary filter where $C(s)=k_{P}+k_{I} / s$. Only in the absence of noise, the convergence of the state estimate can be proved by Lyapunov's direct method. It means some glitches and spikes caused by noise will be introduced to the fusion results through integral.

\section{Extended Kalman Filter}

The Kalman filter addresses the problem of trying to estimate the state vector $\boldsymbol{x} \in \boldsymbol{R}^{n}$ [10]. In the application of fusing multi-sensor data, the input measurements are divided into two groups. One group of measurements comes from the main sensor which will be applied to figure out the priori state estimate $\hat{\boldsymbol{x}}_{k}^{-}$and it can be written as

$$
\hat{\boldsymbol{x}}_{k}^{-}=f\left(\hat{\boldsymbol{x}}_{k-1}, \boldsymbol{u}_{k}, 0\right)
$$

where $\hat{\boldsymbol{x}}_{k-1}$ represents the posteriori state estimate at step $k-1, \boldsymbol{u}_{k} \in \boldsymbol{R}^{l}$ represents the control input at step $k$. Eq. 2 is a non-linear stochastic difference equation.

The other group of measurements comes from the auxiliary sensors that will be regarded as measurement vector $\boldsymbol{z} \in \boldsymbol{R}^{m}$ and the measurement prediction $\hat{z}_{k}$ can be calculated with the priori state estimate $\hat{\boldsymbol{x}}_{k}^{-}$in the form of

$$
\hat{\boldsymbol{z}}_{k}=h\left(\hat{\boldsymbol{x}}_{k}^{-}, 0\right)
$$

It is possible to construct the posteriori state estimate $\hat{\boldsymbol{x}}_{k}$ at step $k$, based on the compensation error of $\hat{\boldsymbol{x}}_{k}^{-}$obtained with the difference value between $\boldsymbol{z}$ and $\hat{\boldsymbol{z}}_{k}$. If Eq. 2 and Eq. 3 are nonlinear, the traditional Kalman filter can do nothing and the EKF is thus developed to deal with the nonlinear 
problem. By means of Taylor expansion, the Jacobian matrix $\boldsymbol{A}$ of partial derivatives of $f$ and Jacobian matrix $\boldsymbol{H}$ of partial derivatives of $h$ with respect to $\boldsymbol{x}$ are given by

$$
\left\{\begin{array}{l}
\boldsymbol{A}_{[i, j]}=\frac{\partial f_{[i]}}{\partial \boldsymbol{x}_{[j]}}\left(\hat{\boldsymbol{x}}_{k-1}, \boldsymbol{u}_{k-1}, 0\right) \\
\boldsymbol{H}_{[i, j]}=\frac{\partial h_{[i]}}{\partial \boldsymbol{x}_{[j]}}\left(\hat{\boldsymbol{x}}_{k}^{-}, 0\right)
\end{array}\right.
$$

The estimate of state $\boldsymbol{x}$ is an iterative process. After each sampling interval, the measurements have to be taken into an iterative formula. The iterative formula, i.e. the discrete extended Kalman filter is expressed as

$$
\left\{\begin{array}{l}
\hat{\boldsymbol{x}}_{k}^{-}=\boldsymbol{f}\left(\hat{\boldsymbol{x}}_{k-1}, \boldsymbol{u}_{k-1}, 0\right) \\
\boldsymbol{P}_{k}^{-}=\boldsymbol{A}_{k} \boldsymbol{P}_{k-1} \boldsymbol{A}_{k}^{T}+\boldsymbol{Q}_{k} \\
\boldsymbol{K}_{k}=\boldsymbol{P}_{k}^{-} \boldsymbol{H}_{k}^{T}\left(\boldsymbol{H}_{k} \boldsymbol{P}_{k}^{-} \boldsymbol{H}_{k}^{T}+\boldsymbol{R}_{k}\right)^{-1} \\
\hat{\boldsymbol{x}}_{k}=\hat{\boldsymbol{x}}_{k}^{-}+\boldsymbol{K}_{k}\left(\boldsymbol{z}_{k}-\boldsymbol{h}\left(\hat{\boldsymbol{x}}_{k}^{-}, 0\right)\right) \\
\boldsymbol{P}_{k}=\left(\boldsymbol{I}-\boldsymbol{K}_{k} \boldsymbol{H}_{k}\right) \boldsymbol{P}_{k}^{-}
\end{array}\right.
$$

where $\boldsymbol{Q}_{k}$ and $\boldsymbol{R}_{k}$ represent the process noise covariance and measurement noise covariance, $\boldsymbol{P}_{k}^{-}$and $\boldsymbol{P}_{k}$ represent priori and posteriori estimate error covariance, the matrix $\boldsymbol{K}_{k}$ is the gain or blending factor that minimizes the posteriori estimate error covariance. The measurement noise covariance $\boldsymbol{R}_{k}$ plays an important role in the performance of final fusion results. If we increase $\boldsymbol{R}_{k}$, the convergence speed of the filter will slow down and the curves of state estimate become smoother, and the effect of measurement vector $\boldsymbol{z}_{k}$ will be reduced. However, we hope that the measurement vector $\boldsymbol{z}_{k}$ can still affect the priori state estimate $\hat{\boldsymbol{x}}_{k}^{-}$effectively when the noise is filtered.

\section{Complementary Filter for Attitude Estimation}

To design a complementary filter for attitude estimation, we define a body fixed coordinate frame, denoted $b$, with $x_{b}$ indicating the front, $y_{b}$ indicating the right, and $z_{b}$ indicating the bottom and a navigation coordinate frame, denoted $n$, with $x_{n}$ indicating the North, $y_{n}$ indicating the East, and $z_{n}$ indicating the ground. The rotation transformation from navigation coordinate frame $n$ to body fixed coordinate frame $b$ can be achieved by a normalized quaternion, i.e. ${ }_{n}^{b} \boldsymbol{q}=\left[\begin{array}{llll}q_{0} & q_{1} & q_{2} & q_{3}\end{array}\right]$. Quaternions are used to represent the orientations which can avoid the singularity problem appeared in the algorithms using Euler angles [11,12].

The measurements of MEMS triaxial gyros, triaxial accelerometers and triaxial magnetometers about the $x, y$ and $z$ axes of the senor frame are respectively arranged into zero-scalar quaternions, i.e. ${ }^{b} \boldsymbol{\Omega}=\left[\begin{array}{llll}0 & \omega_{x} & \omega_{y} & \omega_{z}\end{array}\right],{ }^{b} \boldsymbol{a}=\left[\begin{array}{llll}0 & a_{x} & a_{y} & a_{z}\end{array}\right],{ }^{b} \boldsymbol{m}=\left[\begin{array}{llll}0 & m_{x} & m_{y} & m_{z}\end{array}\right]$ where quaternions ${ }^{b} \boldsymbol{a}$ and ${ }^{b} \boldsymbol{m}$ have been normalized. In addition, an appropriate convention would be to assume that the direction of the gravity ${ }^{n} \boldsymbol{g}$ parallels to the vertical axis $z_{n}$, i.e. ${ }^{n} \boldsymbol{g}=\left[\begin{array}{llll}0 & 0 & 0 & 1\end{array}\right]$ and the earth's magnetic field ${ }^{n} \boldsymbol{b}$ consists of components in horizontal axis $x_{n}$ and vertical axis $z_{n}$ due to the inclination of the field, i.e. ${ }^{n} \boldsymbol{b}=\left[\begin{array}{llll}0 & b_{x} & 0 & b_{y}\end{array}\right]$.

As has been discussed, the drift and static bias of gyros' measurements have a great influence on the quaternions. Therefore, a compensation error $\boldsymbol{e}$ has to be introduced to correct the measurements of 
angular rates. Noting that the correction information comes from accelerometers and magnetometers, the compensation error $\boldsymbol{e}$ is divided into two parts, $\boldsymbol{\xi}$ and $\boldsymbol{\varepsilon}$ for further calculation.

Considering that the measurements of accelerometers are expressed in the body fixed coordinates, the quaternion ${ }^{n} \boldsymbol{g}$ has to be converted to the body fixed coordinate too. The process of calculating error component $\boldsymbol{\xi}$ can be described as

$$
\left\{\begin{array}{l}
{ }^{b} \boldsymbol{g}_{k}={ }_{n}^{b} \hat{\boldsymbol{q}}_{k-1}^{*} \otimes{ }^{n} \boldsymbol{g} \otimes{ }_{n}^{b} \hat{\boldsymbol{q}}_{k-1}=\left[\begin{array}{llll}
0 & g_{x} & g_{y} & g_{z}
\end{array}\right] \\
\boldsymbol{\xi}=\left[\begin{array}{lll}
0 & { }^{b} \boldsymbol{g}^{\prime}{ }_{k} \times{ }^{b} \boldsymbol{a}^{\prime}{ }_{k}
\end{array}\right]
\end{array}\right.
$$

where ${ }^{b} \boldsymbol{g}^{\prime}{ }_{k}=\left[\begin{array}{lll}g_{x} & g_{y} & g_{z}\end{array}\right],{ }^{b} \boldsymbol{a}^{\prime}{ }_{k}=\left[\begin{array}{lll}a_{x} & a_{y} & a_{z}\end{array}\right]$ and operator $\otimes$ denotes a quaternion product.

The earth's magnetic field ${ }^{n} \boldsymbol{b}$ can be calculated with the measurements of magnetometers by

$$
\left\{\begin{array}{l}
{ }^{n} \boldsymbol{h}_{k}={ }_{n}^{b} \hat{\boldsymbol{q}}_{k-1} \otimes{ }^{b} \boldsymbol{m}_{k} \otimes{ }_{n}^{b} \hat{\boldsymbol{q}}_{k-1}^{*}=\left[\begin{array}{llll}
0 & h_{x} & h_{y} & h_{z}
\end{array}\right] \\
{ }^{n} \boldsymbol{b}_{k}=\left[\begin{array}{lllll}
0 & b_{x} & 0 & b_{z}
\end{array}\right]=\left[\begin{array}{llll}
0 & \sqrt{h_{x}^{2}+h_{y}^{2}} & 0 & h_{z}
\end{array}\right]
\end{array}\right.
$$

When the calculation of quaternion ${ }^{n} \boldsymbol{b}$ is completed, the remaining process to calculate the error component $\varepsilon$ are similar to the process of calculating the error component $\boldsymbol{\xi}$, and it can be given by

$$
\left\{\begin{array}{l}
{ }^{b} \boldsymbol{p}_{k}={ }_{n}^{b} \hat{\boldsymbol{q}}_{k-1}^{*} \otimes{ }^{n} \boldsymbol{b}_{k} \otimes{ }_{n}^{b} \hat{\boldsymbol{q}}_{k-1}=\left[\begin{array}{llll}
0 & p_{x} & p_{y} & p_{z}
\end{array}\right] \\
\boldsymbol{\varepsilon}=\left[\begin{array}{ll}
0 & { }^{b} \boldsymbol{m}_{k}^{\prime} \times{ }^{b} \boldsymbol{p}_{k}^{\prime}
\end{array}\right]
\end{array}\right.
$$

where ${ }^{b} \boldsymbol{p}^{\prime}{ }_{k}=\left[\begin{array}{lll}p_{x} & p_{y} & p_{z}\end{array}\right],{ }^{b} \boldsymbol{m}^{\prime}{ }_{k}=\left[\begin{array}{lll}m_{x} & m_{y} & m_{z}\end{array}\right]$. Taking sum of $\boldsymbol{\varepsilon}$ and $\boldsymbol{\xi}$, the final compensation error $\boldsymbol{e}$ can be obtained. Fig. 2 shows the block diagram of the overall operation process of a Mahony passive complementary filter in the application of estimating attitude angles based on quaternions. The function $f\left({ }_{n}^{b} \hat{\boldsymbol{q}}_{k-1},{ }^{n} \boldsymbol{g},{ }^{b} \boldsymbol{a}_{k},{ }^{b} \boldsymbol{m}_{k},{ }^{b} \boldsymbol{b}_{k}\right)$ in Fig. 2 expresses the solution of compensation error $\boldsymbol{e}$ as given above.

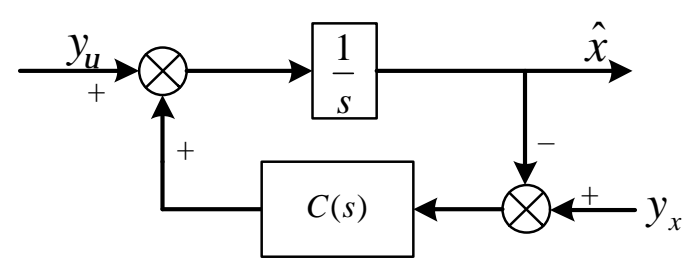

Fig. 1 Block diagram of classical complementary filter

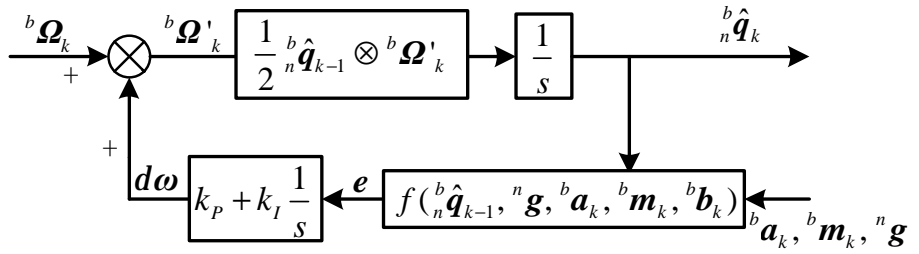

Fig. 2 Block diagram of Mahony filter

\section{Extended Kalman Filter for Attitude Estimation}

Before employing the Extended Kalman filter to estimate attitudes, the state vector $\boldsymbol{x}$ and measurement vector $z$ have to be determined. Since quaternions are adopted to represent the orientations, the state vector $\boldsymbol{x}$ must contain the element of quaternion ${ }_{n}^{b} \boldsymbol{q}$, i.e. $\boldsymbol{x}=\left[\begin{array}{llll}q_{0} & q_{1} & q_{2} & q_{3}\end{array}\right]$.

As described above, the MEMS triaxial gyros are regarded as the main sensor whose measurements will be used to calculate the priori state estimate $\hat{\boldsymbol{x}}_{k}^{-}$. The accelerometers and magnetometers are regarded as the auxiliary sensors to provide measurement vector $z$ which is in the form of 
$z=\left[\begin{array}{ll}{ }^{b} \boldsymbol{a} & { }^{b} \boldsymbol{m}\end{array}\right]$. According to the quaternion differential equations, the exact form of Eq. 2 based on first order Runge-Kutta method is achieved as

$$
\hat{\boldsymbol{x}}_{k}^{-}=\hat{\boldsymbol{x}}_{k-1}+\frac{T}{2}\left[{ }_{n}^{b} \hat{\boldsymbol{q}}_{k-1} \otimes{ }^{b} \boldsymbol{\Omega}^{\prime}{ }_{k}\right]
$$

where ${ }^{b} \boldsymbol{\Omega}^{\prime}{ }_{k}=\left[\begin{array}{llll}0 & \omega_{x}-\omega_{x} & \omega_{y}-\omega_{\Delta y} & \omega_{z}-\omega_{\Delta z}\end{array}\right]$ and $\omega_{\Delta x}, \omega_{\Delta y}, \omega_{\Delta z}$ represent the static bias of gyros which are assumed to be the constants. In order to get the iterative formula as Eq. 5, the measurement prediction vector $\hat{z}_{k}$ must be constructed. Assuming that accelerometers only measure gravity and magnetometers only measure the earth's magnetic field, the exact form of Eq. 3 can be obtained through the convention of ${ }^{n} \boldsymbol{g},{ }^{n} \boldsymbol{b}$ as

$$
\hat{\boldsymbol{z}}_{k}=\left[\begin{array}{c}
{ }^{b} \boldsymbol{g}_{k} \\
{ }^{b} \boldsymbol{p}_{k}
\end{array}\right]=\left[\begin{array}{c}
{ }_{n}^{b} \hat{\boldsymbol{q}}_{k-1}^{*} \otimes{ }^{n} \boldsymbol{g} \otimes{ }_{n}^{b} \hat{\boldsymbol{q}}_{k-1} \\
{ }_{n}^{b} \hat{\boldsymbol{q}}_{k-1}^{*} \otimes{ }^{n} \boldsymbol{b}_{k} \otimes{ }_{n}^{b} \hat{\boldsymbol{q}}_{k-1}
\end{array}\right]
$$

Once the process function $f$ and measurement function $h$ are determined, the orientation quaternion can be determined for each iteration by using the discrete extended Kalman filter as Eq. 5 .

\section{The Proposed Fusion Method for Attitude Estimation}

The proposed fusion method adopts a Mahony passive complementary filter to correct the angular rates measured by gyros. This means the PI controller mentioned above will be applied to compensate the error caused by static bias and drifts, i.e. ${ }^{b} \boldsymbol{\Omega}^{\prime}{ }_{k}=\left[\begin{array}{llll}0 & \omega_{x}-\delta \omega_{x} & \omega_{y}-\delta \omega_{y} & \omega_{z}-\delta \omega_{z}\end{array}\right]$ where $\delta \omega_{x}, \delta \omega_{y}, \delta \omega_{z}$ represent the calculated values of static bias and drifts which are similar to $\omega_{\Delta x}, \omega_{\Delta y}, \omega_{\Delta z}$ appeared in traditional EKF method. The difference between $\delta \omega_{x}, \delta \omega_{y}, \delta \omega_{z}$ and $\omega_{\Delta x}, \omega_{\Delta y}, \omega_{\Delta z}$ lies that $\delta \omega_{x}, \delta \omega_{y}, \delta \omega_{z}$ are varying with time rather than constants. In fact, it is impossible for static bias and drifts of angular rates measured by gyros to be constants due to a variety of factors such as temperature, vibration, collision and so on. The block diagram of the proposed fusion method is described in Fig. 3.

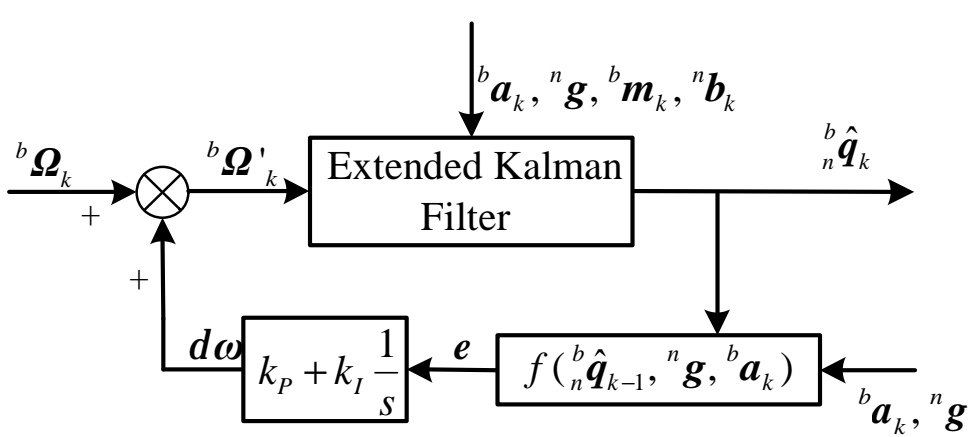

Fig. 3 Block diagram of the proposed fusion method

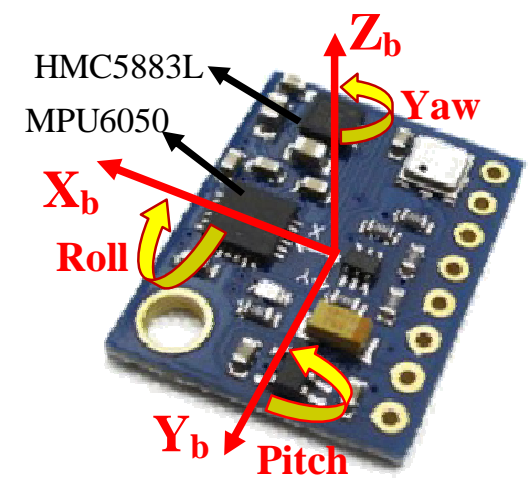

Fig. 4 The MEMS MARG sensors

As shown in Fig. 3, the measurements of accelerometers ${ }^{b} \boldsymbol{a}_{k}$ are used to correct the measurements of gyros ${ }^{b} \boldsymbol{\Omega}_{k}$ with a Mahony passive complementary filter. In other words, in the error components of $\boldsymbol{\xi}$ and $\boldsymbol{\varepsilon}$, only $\boldsymbol{\xi}$ is preserved for the reason that the error component $\boldsymbol{\xi}$ contains the information of pitch and roll angles while the error component $\boldsymbol{\varepsilon}$ is relative to yaw angle. Moreover, the fusion strategy of Mahony passive complementary filter in attitude estimation of rotor UAV does not consider the interaction of each other. If we simply stack the error components, the phenomenon of deviation 
from true value of attitude angles will happen. For the rotor UAV, we emphasize the accuracy of pitch and roll angles. The measurements of gyros ${ }^{b} \boldsymbol{\Omega}_{k}^{\prime}$ corrected by real-time value of $\delta \omega_{x}, \delta \omega_{y}, \delta \omega_{z}$ are fused with the measurements of accelerometers and magnetometers ${ }^{b} \boldsymbol{a}_{k},{ }^{b} \boldsymbol{m}_{k}$ through the discrete EKF. The output of EKF is the quaternion ${ }_{n}^{b} \hat{\boldsymbol{q}}_{k}$, which contains the information of attitude angles of rotor UAV.

\section{Experiment}

A miniature module of MEMS MARG sensors composed of MPU6050 and HMC5883L is shown in Fig. 4. The triaxial accelerometers and triaxial gyros are integrated in MPU6050, and the triaxial magnetometers are integrated in HMC5883L. In fact, the MEMS triaxial accelerometers and triaxial magnetometers constitute a 3D E-Compass. A DSP controller is adopted to fulfill the task of sampling. The collected measurements will be input to PC to do data fusion.

To test the performance of the proposed fusion algorithm, experiments are conducted as follows. Firstly, without using any fusion algorithms, two sets of attitude angles are derived from the measurements of MEMS triaxial gyros and the measurements of 3D E-Compass. Then, taking the measurements of magnetic, angular rate and gravity sensors as inputs, three sets of attitude angles are achieved with three fusion algorithms, i.e., extended Kalman filter, Mahony passive complementary filter and the proposed method. Results of these experiments are shown in Fig. 5, Fig. 6 and Fig 7.

Fig. 5 shows the comparison of attitude angles obtained with non-fusion methods. It is obvious that the curves of roll, pitch, yaw angles obtained from 3D E-Compass are full of glitches and spikes because the accelerometers are susceptible to vibration, acceleration and deceleration, and the magnetometers are susceptible to magnetic noise. In contrast, the curves of roll, pitch, yaw angles obtained from gyros don't exist this problem, but fluctuations of amplitude over time are increased due to the drifts and static bias accumulated through integral.
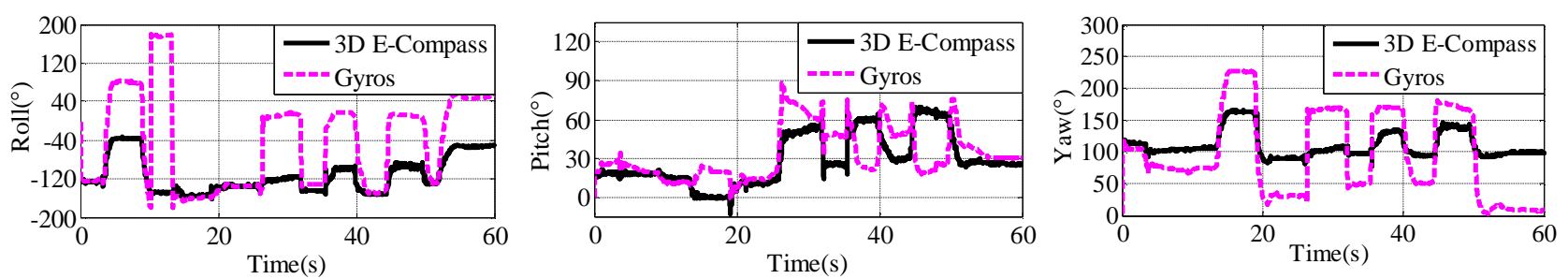

Fig. 5 The comparison of attitude angles obtained with non-fusion methods

Fig. 6 shows the comparison of attitude angles obtained with two fusion algorithms (EKF and Mahony filter) and with non-fusion method (derived from 3D E-Compass). In this way, the curves from accelerometers and magnetometers, i.e. the 3D E-Compass can be used to determine the accuracy of fusion algorithms. It indicates that the extended Kalman filter has higher estimating accuracy than Mahony passive complementary filter, since the simple stack of compensation error components $\xi$ and $\varepsilon$ causes the deviation of final attitude estimation. But in terms of waveforms, the curves of attitude angles from Mahony passive complementary filter are smoother than that of EKF.
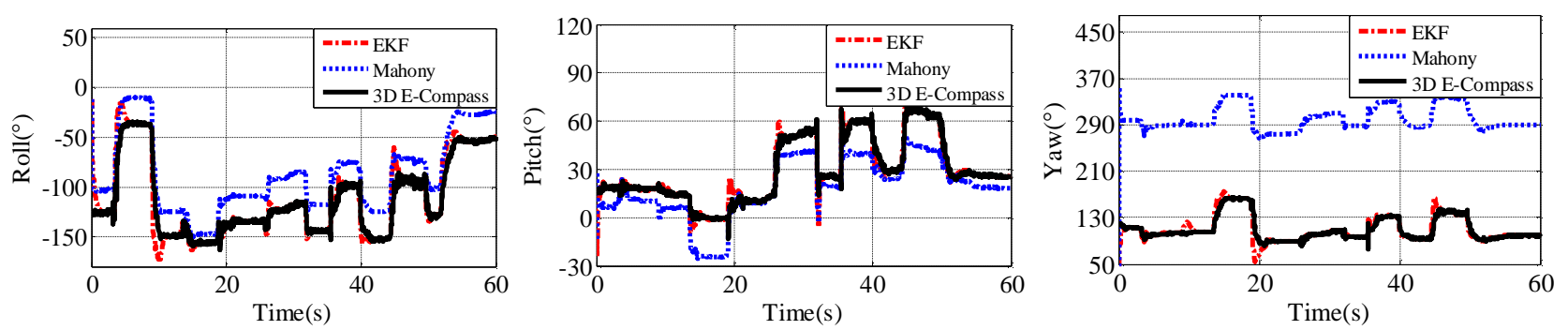

Fig. 6 The comparison of attitude angles obtained with fusion algorithms (EKF and Mahony filter) and with non-fusion method (3D E-Compass) 
Fig. 7 shows the comparison of attitude angles obtained with three fusion algorithms. It can be found that the proposed fusion algorithm has the same accuracy of extended Kalman filter and improves the convergence speed at the same time. In addition, the proposed fusion algorithm also has the capacity of smoothing spikes, which is possessed by Mahony passive complementary filter.
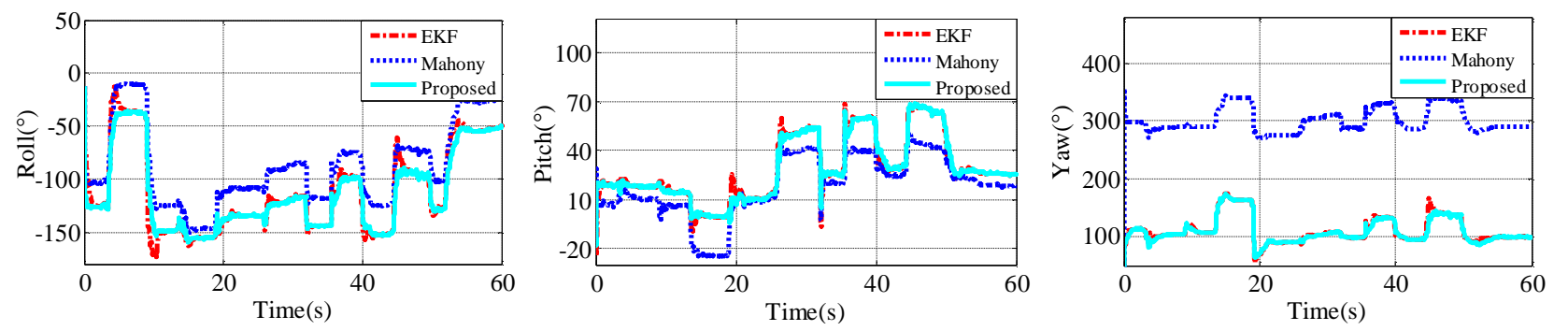

Fig. 7 The comparison of attitude angles obtained with different fusion algorithms

\section{Conclusions}

This paper presents a fusion algorithm combining EKF with complementary filter to estimate the attitude of rotor UAV based on MEMS MARG sensors. The measurements of accelerometers are used to correct the measurements of gyros with a Mahony passive complementary filter. The corrected measurements of gyros are fused with the measurements of accelerometers and magnetometers through a discrete EKF. The output of the discrete EKF is the quaternion, which contains the information of attitude angles of rotor UAV. Results of comparison experiments show that the proposed fusion algorithm can improve the accuracy and reliability, and has the advantages of both EKF and Mahony passive complementary filter. The curves of roll, pitch and yaw angles become smoother as glitches and spikes are reduced, especially the fluctuations caused by drifts and static bias are under control by using the proposed fusion algorithm.

\section{References}

[1] P.D. Groves: Aerospace and Electronic Systems Magazine Vol. 30 (2015), p. 42-69.

[2] Y.C. Lai, S.S. Jan and F.B. Hsiao: Sensors Vol. 10 (2010), p. 2472-2491.

[3] R. Zhu, D. Sun and Z. Zhou, et al: Measurement Vol. 40 (2007), p. 322-328.

[4] S.O. Madgwick, A.J. Harrison and R. Vaidyanathan: IEEE International Conference on Rehabilitation Robotics (ICORR 2011), p. 1-7.

[5] B. Khaleghi, A. Khamis and F.O. Karray, et al: Information Fusion Vol. 14 (2013), p. 28-44.

[6] D. Gebre-Egziabher, R.C. Hayward, J.D. Powell: Aerospace and Electronic Systems Vol. 40 (2004), p. 627-649.

[7] A.M. Sabatini: Biomedical Engineering Vol. 53 (2006), p. 1346-1356.

[8] R. Kalman: Transaction of the ASME-Journal of Basic Engineering Vol. 82d (1960), p. 35-45.

[9] R. Mahony, T. Hamel and J.M. Pflimlin: Automatic Control Vol. 53 ( 2008), p. 1203-1218.

[10] G. Welch, G. Bishop: University of North Carolina: Chapel Hill, North Carolina, US (2006).

[11] J. Diebel: Matrix Vol. 58 (2006), p. 15-16.

[12]B. Delporte, L. Perroton and T. Grandpierre, et al: Sensors and Transducers Journal Vol. 14 (2012), p 32-47. 